\title{
Contributo da formação musical no desempenho académico e cognitivo de crianças e adolescentes: Uma revisão sistemática
}

\section{RESUMO}

Esta revisão sistemática teve como objetivo analisar a literatura recente acerca da influência que o estudo da música pode exercer no desempenho académico em geral e na matemática; nas funções cognitivas; e na plasticidade cerebral. A revisão inclui estudos publicados entre 2007 e 2018, nas bases de dados PubMed e Complementary Index, Academic Search Complete, Education Source, Psychology and Behavioral Sciences Collection, Science Direct e PsycArticles utilizando o descritor musical training, combinado com descritores ligados ao desempenho académico em geral e na matemática - academic achievement, mathematics e academic development - e ao desenvolvimento cognitivo - brain development e cognitive development. Os estudos foram selecionados de acordo com os seguintes critérios: estudos (i) publicados em jornais científicos revistos por pares, (ii) com crianças e adolescentes até aos 18 anos, (iii) que incluíram o treino musical nas suas componentes teórica e/ou instrumental. A relação entre a formação musical e o desempenho académico em geral e a matemática mostrou-se inconsistente, não existindo consenso na literatura acerca dos benefícios da primeira sobre a segunda. Foram encontrados benefícios cognitivos e evidências para a plasticidade cerebral estrutural, induzida pelo treino musical na primeira infância, tendo em consideração as diferenças encontradas no volume de massa cinzenta.

Palavras-chave: Formação musical; Desempenho académico; Competências matemáticas; Desenvolvimento cognitivo.

\section{INTRODUÇÃO}

A relação entre a música e a matemática tem sido considerada há milhares de anos (Sanders, 2012). Nos ensinamentos de Pitágoras (cerca de 500 a.C.) e dos seus seguidores, a música e a aritmética não eram disciplinas separadas, os números eram considerados a chave de todo o universo espiritual e físico. 0 sistema dos sons e ritmos musicais, sendo regido pelo número, exemplificava a harmonia do cosmos e correspondia a essa harmonia (Grout \& Palisca,
Susana Azevedo ${ }^{i}$

Universidade Católica

Portuguesa, Portugal

Joana Rato ${ }^{\text {i }}$

Universidade Católica Portuguesa, Portugal

Alexandre Castro Caldasiii Universidade Católica Portuguesa, Portugal 
2014). No tempo dos gregos antigos, a matemática e a música estavam fortemente conectadas. A música era considerada uma disciplina estritamente matemática, lidando com relações numéricas e proporções (Beer, 2005).

A música, no contexto do Quadrivium (curriculum da escola pitagórica), era colocada ao mesmo nível da aritmética, geometria e astronomia, constituindo as artes matemáticas. Essa interpretação negligenciou os aspetos criativos da performance musical. A música era a ciência do som e da harmonia (Beer, 2005).

Nos anos 90, um estudo de Rauscher et al. (1994) fez renascer o interesse pelos benefícios não-musicais decorrentes da exposição musical quando indicou que uma breve exposição (10 min.) a uma sonata de Mozart gerava um aumento a curto prazo das capacidades de raciocínio espacial. É nesta altura que surge o designado "Efeito Mozart", que deu fundamento à construção de diversas crenças relativamente à relação existente entre o domínio da música e a cognição. Jones e Zigler (2002) mencionam um estudo de Rauscher et al. (1997) realizado com crianças em idade pré-escolar (entre os 3 e os 5 anos) que tiveram aulas particulares de piano de 10 minutos por dia durante 6 meses. Os investigadores verificaram que o grupo de piano apresentou melhores resultados numa tarefa de montagem de objetos que requeria competências espácio-temporais, do Weschler Preschool and Primary School Test of Intelligence (WPPSI), comparativamente às crianças que beneficiaram de aulas de informática, canto ou sem aulas. Perante os resultados obtidos, os investigadores descreveram estas mudanças na capacidade espácio-temporal como "de longo prazo", uma vez que eram igualmente evidentes tanto nas crianças que foram testadas em menos de um dia após a última aula, como naquelas que foram testadas um dia após o término. No entanto, a definição excecionalmente limitada de "a longo prazo" aplicada a um contexto de 10 minutos e um ou dois dias tornou a conclusão puramente especulativa.

Jones e Zigler (2002) citam ainda outros investigadores que tentaram replicar o "Efeito Mozart", para distinguir a superioridade induzida pela música em testes e subtestes cognitivos, sem sucesso (cf. Kenealy \& Monseth, 1994; Newman et al., 1995; Steele et al., 1997, 1999; Stough et al., 1994; Wilson \& Brown, 1997). Posteriormente, o "Efeito Mozart" foi muito criticado, já que foram várias as limitações apontadas ao método de estudo utilizado. Já O'Hanlon (1981) tinha salientado que os estados de humor poderiam influenciar o desempenho de tarefas quando o indivíduo, de alguma forma, era exposto a um estímulo monótono. Assim, este efeito poderia ter surgido a partir da existência de diferenças de humor ou excitação, e não da exposição a Mozart (Schellenberg, 2001).

Mais recentemente o estudo da música ganhou interesse adicional na educação, tendo em consideração estudos neurocientíficos que demonstram efeitos positivos no desenvolvimento do cérebro e, concomitantemente, no desempenho académico. Alguns autores, como Beer (2005), Kravanja (2008) e Wright (2009), referem que existem numerosas ligações entre a música e a matemática, tais como as relações numéricas, as proporções, os números inteiros, os logaritmos, as operações aritméticas, a trigonometria e a geometria, que se encontram relacionados com elementos musicais como a melodia, o ritmo, os intervalos, as escalas e a harmonia. 
Até agora, diversos estudos têm sido conduzidos abordando os efeitos que o estudo da música pode exercer no desenvolvimento cognitivo e, concomitantemente, no desempenho académico. No entanto, os resultados desses estudos têm sido considerados inconclusivos ou contraditórios. Para além disso, nenhum dos estudos focou os efeitos musicais nas competências matemáticas (Catterall et al., 1999; Costa-Giomi, 1999; Fitzpatrick, 2006; Moreno et al., 2011; Morrison, 1994; Schellenberg, 2011; Schlaug et al., 2005; Schneider \& Klotz, 2000; Southgate \& Roscigno, 2009; Wetter et al., 2009). Porém, os trabalhos de Miendlarzewska e Trost (2014), Ramalho (2014), Luís (2013) e Moura (2012) apresentaram uma perspetiva diferente.

Por um lado, atribuíram à formação musical efeitos de transferência diretos e indiretos, preparando uma base para uma variedade de competências que promoviam o desenvolvimento cognitivo. Por exemplo, as crianças que aprendem música apresentam uma melhor memória verbal, capacidade de aprender uma segunda língua, capacidade de leitura e funções executivas. 0 entrainment do ritmo foi explicitamente proposto como o mecanismo que apoia o desenvolvimento de funções executivas para efeitos de treinamento da música e da dança (Miendlarzewska \& Trost, 2014).

Quanto aos estudos conduzidos a nível nacional, Ramalho (2014) estudou os efeitos, ao nível das funções executivas e da reprodução de estruturas rítmicas de crianças, de uma metodologia de iniciação musical aplicada em crianças em idade pré-escolar, num período de 3 anos. Fizeram parte do primeiro grupo de intervenção 21 crianças e do segundo grupo 19, com um grupo de controlo de 31 crianças. Os resultados revelaram que a iniciação musical teve impacto significativo e positivo nas funções executivas e no desenvolvimento da capacidade rítmica. Para além disso, quanto maior for tempo de aprendizagem, maior é o desenvolvimento, quer das funções executivas, quer da capacidade rítmica. Concluiu-se que 1 ano após o término da intervenção, não existiu retrocesso nos benefícios obtidos a partir da aprendizagem inicial de música.

No estudo longitudinal conduzido por Luís (2013), foram estudados alunos desde o $7^{\circ}$ ao $9^{\circ}$ ano, quanto à influência da aprendizagem musical no próprio desenvolvimento musical, para além de observar o efeito de preditores no desempenho matemático (nível socioeconómico, inteligência e variáveis cognitivo-motivacionais). A amostra abrangeu 112 alunos do ensino especializado de música e do ensino regular. Os alunos foram avaliados a partir da avaliação sumativa na disciplina de matemática e dos resultados no exame nacional de matemática. Os resultados revelaram que os alunos que frequentavam o ensino formal de música apresentaram um desempenho matemático superior relativamente aos alunos sem este tipo de ensino, sustentando também a hipótese de que o número de anos de aprendizagem musical contribui para o aumento do desempenho matemático.

Já Moura (2012), analisou os contributos da participação em projetos artísticos e musicais (Clubes de Música) no desempenho académico de alunos que frequentavam o $2^{\circ}$ ciclo do Ensino Básico. A amostra era composta por 379 alunos e, destes, 77 participavam em Clubes de Música. Os resultados revelaram uma relação positiva entre a frequência de atividades extracurriculares (frequência do Clube de Música) e o desempenho académico no geral e 
individualmente em Língua Portuguesa, Matemática e Educação Musical. Foi observada, simultaneamente, uma relação também positiva entre a frequência do Clube de Música e o desenvolvimento de competências musicais.

Por sua vez, o estudo de Pereira (2012) menciona que a música enquanto atividade extracurricular não explica, só por si, outro tipo de benefício cognitivo não musical. Neste estudo foram analisadas as diferenças cognitivas entre o estudante profissional de música e o estudante de ensino regular, tendo em consideração os dois ambientes distintos em que estão inseridos. A amostra foi composta por 190 alunos, com idades compreendidas entre os 12 e os 22 anos. Destes, 119 frequentavam o ensino regular e 71 o ensino profissional. Os resultados revelaram uma relação positiva entre a música e as capacidades cognitivas melhoradas (abstrata, numérica, espacial e mecânica), à exceção do domínio verbal, em ambiente profissional. 0 tempo de experiência de ensino musical foi também associado a melhores capacidades cognitivas, sendo que à medida que os alunos vão evoluindo nos seus estudos musicais maior é a hipótese de melhorarem significativamente as suas capacidades cognitivas.

Assim, esta revisão visa reunir os estudos presentes nas bases de dados internacionais para identificar e refletir sobre a variação dos resultados publicados, nos últimos anos, no campo da formação musical e o efeito de transferência indireta no desempenho académico e cognitivo, bem como diferenças cerebrais estruturais e funcionais entre músicos e não-músicos.

\section{MATERIAL E MÉTODOS}

Conduzimos uma revisão sistemática bibliográfica com recurso às seguintes bases de dados online: PubMed, Complementary Index, Academic Search Complete, Education Source, Psychology and Behavioral Sciences Collection, Science Direct e PsycArticles. Utilizámos um descritor relacionado com a formação musical, para o qual utilizámos o termo musical training, combinado com descritores ligados ao desempenho académico, em geral e na matemática, com os termos academic achievement, mathematics e academic development, e ao desenvolvimento cognitivo, com os termos brain development e cognitive development. Foi estabelecido um período de 11 anos para a pesquisa, compreendendo trabalhos publicados entre 2007 e 2018, escritos em língua inglesa. A análise inicial implicou uma classificação dos artigos, com base numa seleção mais detalhada de acordo com critérios de inclusão e exclusão, por forma a identificar os estudos relevantes de acordo com o objeto de estudo proposto. Foram incluídos estudos (i) publicados em jornais científicos revistos por pares (peer-reviewed), (ii) realizados com crianças e adolescentes até aos 18 anos, (iii) que incluíram o treino musical nas suas componentes teórica e/ou instrumental. Foram excluídos os seguintes: (i) estudos que utilizaram currículos integrados (p.e., aulas de música e leitura na mesma intervenção); (ii) estudos em que não existiu um grupo de controlo; (iii) estudos com adultos; e (iv) estudos com crianças e adolescentes com dificuldade de aprendizagem específica ou condição clínica. Após este processo de seleção, foi feita uma leitura integral dos trabalhos escolhidos procedendo-se a uma análise detalhada. 
Com a pesquisa realizada nas sete bases de dados, utilizando os descritores anteriormente referidos, foram encontrados 1934 trabalhos. As referências duplicadas foram eliminadas e o número de artigos identificados reduziu para 1885 (Figura 1 ).

\section{Figura 1 \\ Método de Seleção dos Estudos Incluídos nesta Revisão}

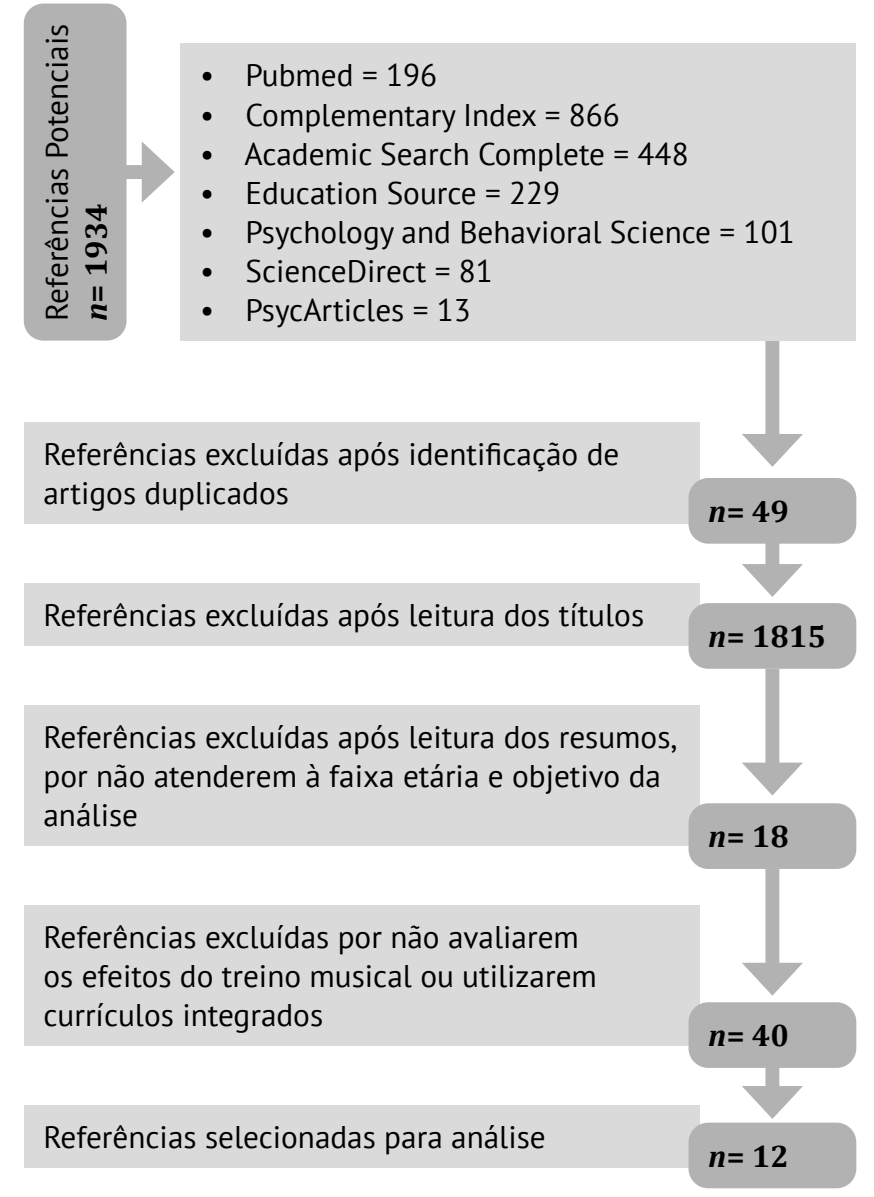

Posteriormente, foram analisados os títulos e/ou resumos desses artigos, tendo sido excluídos: (i) artigos que não avaliavam especificamente os efeitos do treino musical nas suas componentes teórica e/ou instrumental na matemática ou no desempenho académico em geral ou nas funções cognitivas ou na plasticidade cerebral; (ii) estudos com crianças e adolescentes com dificuldades de aprendizagem específica ou condição clínica; (iii) estudos com adultos; (iv) estudos em que foram utilizados currículos integrados; (v) estudos em que não existiu um grupo de controlo. A partir desta seleção inicial, foram selecionados 40 artigos para uma leitura completa. No final, foram selecionados 12 estudos que cumpriam na totalidade os critérios estabelecidos previamente. 


\section{RESULTADOS}

Da análise aos 12 estudos selecionados, verificou-se que estes foram conduzidos em oito países e publicados em 11 jornais revistos por pares (Tabela 1). No total, os estudos abrangeram 2541 crianças e adolescentes, com idades entre os 5 e os 17 anos, as quais foram avaliadas relativamente (i) ao desenvolvimento do raciocínio matemático; e/ou (ii) no desempenho académico em geral; e/ou (iii) nas funções cognitivas (memória e atenção); e/ou (iv) na plasticidade cerebral. A maior parte dos autores diferenciou o género, permitindo observar que, no total da amostra, há uma maior percentagem de crianças e adolescentes do género masculino (51\%) do que do género feminino.

Todos os estudos são empíricos, cinco longitudinais, um transversal, quatro prospetivos, um retrospetivo e um de ensaio.

Tabela 1

Estudos Selecionados

\begin{tabular}{|c|c|c|c|c|c|c|c|c|c|}
\hline \multirow[t]{2}{*}{ Autores } & \multirow[t]{2}{*}{ Ano } & \multirow[t]{2}{*}{ País } & \multirow[t]{2}{*}{ Jornais } & \multirow[t]{2}{*}{ (n) } & \multicolumn{2}{|c|}{ Género } & \multicolumn{2}{|l|}{ Idade } & \multirow{2}{*}{$\begin{array}{l}\text { Tipo de } \\
\text { Estudo }\end{array}$} \\
\hline & & & & & $M$ & $\mathbf{F}$ & $\begin{array}{l}\text { Média } \\
\text { (DP) }\end{array}$ & Intervalo & \\
\hline $\begin{array}{l}\text { D'Souza } \\
\& \\
\text { Wiseheart }\end{array}$ & 2018 & Canadá & $\begin{array}{l}\text { Archives of } \\
\text { Scientific } \\
\text { Psychology }\end{array}$ & 75 & 31 & 44 & & $6-9$ & Transversal \\
\hline Guo et al. & 2018 & Japão & $\begin{array}{l}\text { Frontiers in } \\
\text { Psychology }\end{array}$ & 40 & 22 & 18 & $\begin{array}{l}7.49 \\
(0.58)\end{array}$ & & $\begin{array}{l}\text { Piloto } \\
\text { exploratório } \\
\text { (Ensaio } \\
\text { clínico } \\
\text { aleatório) }\end{array}$ \\
\hline $\begin{array}{l}\text { Jaschke } \\
\text { et al. }\end{array}$ & 2018 & $\begin{array}{l}\text { Reino } \\
\text { Unido }\end{array}$ & $\begin{array}{l}\text { Frontiers in } \\
\text { Psychology }\end{array}$ & 146 & NA & NA & $\begin{array}{l}6.4 \\
(0.65)\end{array}$ & & Longitudinal \\
\hline $\begin{array}{l}\text { Hallam \& } \\
\text { Rogers }\end{array}$ & 2016 & $\begin{array}{l}\text { Reino } \\
\text { Unido }\end{array}$ & $\begin{array}{l}\text { British } \\
\text { Journal } \\
\text { of Music } \\
\text { Education }\end{array}$ & 608 & NA & NA & & $11-16$ & Retrospetivo \\
\hline $\begin{array}{l}\text { Nutley } \\
\text { et al. }\end{array}$ & 2014 & Suécia & $\begin{array}{l}\text { Frontiers } \\
\text { in Human } \\
\text { Neuroscience }\end{array}$ & 339 & 171 & 168 & & $6-25$ & Longitudinal \\
\hline $\begin{array}{l}\text { Roden } \\
\text { et al. }\end{array}$ & 2014 & Alemanha & $\begin{array}{l}\text { Applied } \\
\text { Cognitive } \\
\text { Psychology }\end{array}$ & 345 & 190 & 155 & $\begin{array}{l}7.87 \\
(0.82)\end{array}$ & & Longitudinal \\
\hline $\begin{array}{l}\text { Yang } \\
\text { et al. }\end{array}$ & 2014 & China & $\begin{array}{l}\text { Scientific } \\
\text { Reports }\end{array}$ & 250 & 128 & 122 & $\begin{array}{l}6.6 \\
(4.30) \\
\end{array}$ & & Longitudinal \\
\hline $\begin{array}{l}\text { Zuk } \\
\text { et al }\end{array}$ & 2014 & EUA & PLOS ONE & 27 & 11 & 16 & $\begin{array}{l}10.9 \\
(1.2) \\
\end{array}$ & & Prospetivo \\
\hline $\begin{array}{l}\text { Cabanac } \\
\text { et al. }\end{array}$ & 2013 & Canadá & $\begin{array}{l}\text { Behavioural } \\
\text { Brain } \\
\text { Research }\end{array}$ & 560 & NA & NA & & $14-17$ & Prospetivo \\
\hline $\begin{array}{l}\text { Hyde } \\
\text { et al. }\end{array}$ & 2009 & EUA & $\begin{array}{l}\text { The } \\
\text { Journal of } \\
\text { Neuroscience }\end{array}$ & 31 & 15 & 16 & $\begin{array}{l}6.32 \\
(0.82)\end{array}$ & & Longitudinal \\
\hline $\begin{array}{l}\text { Forgeard } \\
\text { et al. }\end{array}$ & 2008 & EUA & PLOS ONE & 59 & 30 & 29 & $\begin{array}{l}9.96 \\
(0.74)\end{array}$ & & Prospetivo \\
\hline
\end{tabular}


Nos 12 estudos, os resultados obtidos em crianças com formação musical (teórica e/ou instrumental) foram comparados a resultados de crianças sem formação musical (grupos de controlo), emparelhados por várias variáveis. É de salientar a existência de mais artigos no ano de 2014 e o facto de haver um período de 2 anos, entre 2010 e 2012, em que não foram encontrados estudos que se enquadrassem na revisão em análise.

As principais características de cada um dos estudos incluídos nesta revisão são apresentados na Tabela 2.

Tabela 2

Principais Características e Resultados dos Estudos Incluídos

\begin{tabular}{|c|c|c|c|c|c|}
\hline \multirow[t]{2}{*}{ Estudo } & \multicolumn{2}{|c|}{$\begin{array}{l}\text { Participantes } \\
(n)\end{array}$} & \multirow[t]{2}{*}{ Objetivo do estudo } & \multirow[t]{2}{*}{ Medidas de Avaliação } & \multirow{2}{*}{$\begin{array}{l}\text { Influência da Formação Musical } \\
\text { nos Objetivos em Estudo }\end{array}$} \\
\hline & GE & $\mathrm{GC}$ & & & \\
\hline $\begin{array}{l}\text { D' Souza \& } \\
\text { Wiseheart } \\
\text { (2018) }\end{array}$ & 50 & 25 & $\begin{array}{l}\text { Investigar se a } \\
\text { aprendizagem musical } \\
\text { tem influência na } \\
\text { cognição. }\end{array}$ & $\begin{array}{l}\text { Peabody Picture } \\
\text { Vocabulary (PPVT); K-BIT } \\
\text { Matrices; Digit Span } \\
\text { Forward; Digit Span } \\
\text { Backward; } \\
\text { Self-Ordered Pointing } \\
\text { Task; Flanker; Stroop; } \\
\text { Color-Shape; Quantity- } \\
\text { Identify; Box Completion; } \\
\text { Symbol Copy; Digit } \\
\text { Symbol Substitution. }\end{array}$ & $\begin{array}{l}\text { Não foram observados efeitos no } \\
\text { desempenho cognitivo no grupo de } \\
\text { música em comparação com o grupo de } \\
\text { dança ou grupo de controlo. Também } \\
\text { não foram encontrados efeitos nas } \\
\text { competências cognitivas relacionadas } \\
\text { com a aprendizagem musical (memória } \\
\text { de trabalho, controlo de interferência, } \\
\text { troca de tarefas, velocidade de } \\
\text { processamento, vocabulário recetivo } \\
\text { ou inteligência não verbal após } \\
\text { o experimento). As competências } \\
\text { cognitivas essenciais (funções executivas, } \\
\text { velocidade de processamento) ou } \\
\text { mecanismos de aprendizagem } \\
\text { básicos (coordenação multimodal e } \\
\text { entrainment do ritmo) não funcionam } \\
\text { como intermediários para um benefício } \\
\text { cognitivo. Pelo facto de não terem sido } \\
\text { observadas diferenças significativas nos } \\
\text { grupos avaliados, este estudo conclui } \\
\text { que não há benefícios exclusivos do } \\
\text { treino musical a curto prazo. }\end{array}$ \\
\hline $\begin{array}{l}\text { Guo et al. } \\
\text { (2018) }\end{array}$ & 20 & 20 & $\begin{array}{l}\text { Investigar o efeito } \\
\text { de um programa de } \\
\text { prática instrumental de } \\
6 \text { semanas nas funções } \\
\text { executivas de crianças. }\end{array}$ & $\begin{array}{l}\text { Wechsler Intelligence } \\
\text { Scale for Children (WISC- } \\
\text { IV); Teste de RAN. }\end{array}$ & $\begin{array}{l}\text { Foi sugerido que tocar instrumentos } \\
\text { musicais pode ser benéfico para } \\
\text { melhorar a memória de trabalho a curto } \\
\text { prazo. No entanto, não foi encontrada } \\
\text { nenhuma influência cognitiva } \\
\text { significativa no desempenho nas tarefas } \\
\text { que avaliaram a capacidade verbal, } \\
\text { inibição e velocidade de processamento. }\end{array}$ \\
\hline $\begin{array}{l}\text { Jaschke et } \\
\text { al. (2018) }\end{array}$ & 109 & 37 & $\begin{array}{l}\text { Avaliar se as } \\
\text { aulas de música } \\
\text { estruturadas podem } \\
\text { afetar subfunções } \\
\text { executivas adjacentes } \\
\text { ao desempenho } \\
\text { académico. }\end{array}$ & $\begin{array}{l}\text { Tower of London (TOL); } \\
\text { Klingberg Short Term } \\
\text { and Working Memory } \\
\text { Task; Go/No-Go Task; } \\
\text { Wechsler Intelligence } \\
\text { Scale for Children (sub- } \\
\text { tests I - Information, III } \\
\text { - Similarities, V - Verbal } \\
\text { Compreension e VII - } \\
\text { Aritmética); Testes da } \\
\text { National Pupil Monitoring } \\
\text { System (CITO); Music } \\
\text { Intervention. }\end{array}$ & $\begin{array}{l}\text { Foi identificada uma influência positiva } \\
\text { da educação musical a longo prazo } \\
\text { nas competências cognitivas, como } \\
\text { o planeamento e inibição. Foram } \\
\text { registadas simultâneas melhorias nas } \\
\text { tarefas de memória visuoespacial. Para } \\
\text { além disso, foi observado um efeito } \\
\text { de transferência indireta da educação } \\
\text { musical no desempenho académico } \\
\text { mediado por subfunções executivas. }\end{array}$ \\
\hline
\end{tabular}




\begin{tabular}{|c|c|c|c|c|c|}
\hline \multirow[t]{2}{*}{ Estudo } & \multicolumn{2}{|c|}{$\begin{array}{l}\text { Participantes } \\
(n)\end{array}$} & \multirow[t]{2}{*}{ Objetivo do estudo } & \multirow[t]{2}{*}{ Medidas de Avaliação } & \multirow{2}{*}{$\begin{array}{l}\text { Influência da Formação Musical } \\
\text { nos Objetivos em Estudo }\end{array}$} \\
\hline & GE & GC & & & \\
\hline $\begin{array}{l}\text { Hallam \& } \\
\text { Rogers } \\
\text { (2016) }\end{array}$ & 115 & 493 & $\begin{array}{l}\text { Verificar se os } \\
\text { estudantes que } \\
\text { aprendem a tocar um } \\
\text { instrumento musical } \\
\text { têm um melhor } \\
\text { desempenho nos } \\
\text { exames nacionais do } \\
\text { Reino Unido. }\end{array}$ & $\begin{array}{l}\text { Testes académicos } \\
\text { e exames nacionais } \\
\text { nas disciplinas de } \\
\text { Inglês, Matemática e } \\
\text { desempenho global. }\end{array}$ & $\begin{array}{l}\text { Os jovens instrumentistas mostraram } \\
\text { melhor progresso e melhores } \\
\text { resultados académicos do que os não } \\
\text { instrumentistas, com maior impacto } \\
\text { naqueles que tocavam há mais tempo } \\
(4-5 \text { anos). }\end{array}$ \\
\hline $\begin{array}{l}\text { Nutley et } \\
\text { al. } \\
\text { (2014) }\end{array}$ & 280 & 59 & $\begin{array}{l}\text { Determinar se o treino } \\
\text { musical poderá estar } \\
\text { associado: (1) a efeitos } \\
\text { positivos na memória } \\
\text { de trabalho, velocidade } \\
\text { de processamento } \\
\text { e capacidade de } \\
\text { raciocínio, bem como } \\
\text { nas competências } \\
\text { de matemática e } \\
\text { leitura ao longo dos } \\
\text { períodos; (2) com a } \\
\text { quantidade de prática } \\
\text { e a magnitude dos } \\
\text { benefícios cognitivos } \\
\text { e académicos; (3) a } \\
\text { diferenças estruturais } \\
\text { no cérebro entre } \\
\text { músicos e não músicos } \\
\text { em três momentos. }\end{array}$ & $\begin{array}{l}\text { Automated Working } \\
\text { Memory Assessment } \\
\text { (AWMA); } \\
\text { Raven's Advanced } \\
\text { Progressive Matrices; } \\
\text { Trends in Mathematics } \\
\text { and Science Study } \\
\text { (TIMSS) e Basic Number } \\
\text { Screening Test; } \\
\text { Progress in International } \\
\text { Reading Literacy Trend } \\
\text { Study (PIRLS 2001 T) } \\
\text { e The International } \\
\text { Associations Association } \\
\text { for the Evaluation of } \\
\text { Education Achievement } \\
\text { Reading Literacy Study } \\
\text { 1991; } \\
\text { Magnetic Resonance } \\
\text { Imaging (MRI). }\end{array}$ & $\begin{array}{l}\text { A prática musical afeta positivamente } \\
\text { o desenvolvimento da memória de } \\
\text { trabalho e suporta a importância da } \\
\text { prática para o desenvolvimento da } \\
\text { memória de trabalho durante a infância } \\
\text { e adolescência. } \\
\text { Foram encontradas diferenças } \\
\text { significativas no volume de massa } \\
\text { cinzenta, no lobo temporal situado } \\
\text { principalmente nos giros fusiformes } \\
\text { temporais e occipitais inferiores e na } \\
\text { ínsula, caudado e putâmen. }\end{array}$ \\
\hline $\begin{array}{l}\text { Roden et } \\
\text { al. } \\
\text { (2014) }\end{array}$ & 192 & 153 & $\begin{array}{l}\text { Investigar os efeitos } \\
\text { a longo prazo de } \\
\text { um programa de } \\
\text { música instrumental, } \\
\text { em sala de aula, nas } \\
\text { capacidades cognitivas } \\
\text { em crianças em idade } \\
\text { escolar. } \\
\text { Especificamente, } \\
\text { nas competências } \\
\text { cognitivas musicais: } \\
\text { atenção visual, } \\
\text { velocidade de } \\
\text { processamento e de } \\
\text { audição musical, em } \\
\text { três momentos ao } \\
\text { longo de um período } \\
\text { de } 18 \text { meses. }\end{array}$ & $\begin{array}{l}\text { Thorndike's Cognitive } \\
\text { Abilities Test, Primary II/ } \\
\text { Form } 1 \text { and 2; } \\
\text { d2 test of attention; } \\
\text { Zahlen-Verbindungs-Test. }\end{array}$ & $\begin{array}{l}\text { As crianças que receberam treino } \\
\text { musical instrumental apresentaram } \\
\text { aumentos significativos nas } \\
\text { competências musicais e velocidade } \\
\text { de processamento, da segunda para a } \\
\text { terceira avaliação, e um aumento das } \\
\text { capacidades rítmicas, da primeira para a } \\
\text { segunda avaliação. } \\
\text { Embora as crianças com treino musical } \\
\text { tenham mostrado melhorias tanto na } \\
\text { velocidade de processamento quanto na } \\
\text { atenção visual ao longo do tempo, elas } \\
\text { apenas tiveram uma pequena vantagem } \\
\text { na primeira e uma clara desvantagem } \\
\text { na última medida em comparação } \\
\text { com as crianças do grupo de controlo } \\
\text { que receberam formação em ciências } \\
\text { naturais. }\end{array}$ \\
\hline $\begin{array}{l}\text { Yang et al. } \\
\text { (2014) }\end{array}$ & 77 & 173 & $\begin{array}{l}\text { Analisar se o treino } \\
\text { musical está relacionado } \\
\text { com um melhor } \\
\text { desempenho académico } \\
\text { na L1 - Chinês, na L2 - } \\
\text { Inglês, e em Matemática. }\end{array}$ & $\begin{array}{l}\text { Testes padronizados } \\
\text { em L1, L2 e Matemática } \\
\text { (aritmética, geometria e } \\
\text { álgebra); } \\
\text { Treino formal de pintura; } \\
\text { Teste musical: pitch na } \\
\text { música, representação } \\
\text { melódica, canto e teoria } \\
\text { musical; } \\
\text { Acompanhamento } \\
\text { extracurricular na L1, L2 } \\
\text { e Matemática; } \\
\text { Raven IQ test. }\end{array}$ & $\begin{array}{l}\text { As crianças com formação musical } \\
\text { superaram as crianças sem formação } \\
\text { musical apenas no desempenho musical } \\
\text { e no desenvolvimento da segunda } \\
\text { língua (Inglês). Além disso, embora } \\
\text { a formação musical parecesse estar } \\
\text { correlacionada com o desenvolvimento } \\
\text { académico final das crianças na L1 } \\
\text { (Chinês), L2 (Inglês) e Matemática, não } \\
\text { contribuiu de forma independente } \\
\text { para o desenvolvimento da L1 ou } \\
\text { competências matemáticas. }\end{array}$ \\
\hline
\end{tabular}




\begin{tabular}{|c|c|c|c|c|c|}
\hline \multirow[t]{2}{*}{ Estudo } & \multicolumn{2}{|c|}{$\begin{array}{l}\text { Participantes } \\
(n)\end{array}$} & \multirow[t]{2}{*}{ Objetivo do estudo } & \multirow[t]{2}{*}{ Medidas de Avaliação } & \multirow{2}{*}{$\begin{array}{l}\text { Influência da Formação Musical } \\
\text { nos Objetivos em Estudo }\end{array}$} \\
\hline & GE & GC & & & \\
\hline $\begin{array}{l}\text { Zuk et al. } \\
\text { (2014) }\end{array}$ & 15 & 12 & $\begin{array}{l}\text { Investigar a relação } \\
\text { entre o treino musical } \\
\text { e as funções executivas } \\
\text { (FE). }\end{array}$ & $\begin{array}{l}\text { Delis-Kaplan Executive } \\
\text { Function System } \\
\text { (subtestes): Trail Making; } \\
\text { Wechsler Intelligence } \\
\text { Scale for Children IV } \\
\text { (WISC-IV); } \\
\text { Kaufman Brief Intelligence } \\
\text { Test (KBIT); } \\
\text { fMRI set-shifting task. }\end{array}$ & $\begin{array}{l}\text { Crianças treinadas musicalmente } \\
\text { mostraram melhor desempenho do que } \\
\text { crianças não treinadas nas medidas } \\
\text { de flexibilidade cognitiva (fluência } \\
\text { verbal e Trail Making) e velocidade } \\
\text { de processamento, e ativação } \\
\text { significativamente maior na pré-Área } \\
\text { Motora Suplementar / Área Motora } \\
\text { Suplementar e Córtex pré-frontal } \\
\text { ventrolateral, durante a representação } \\
\text { de regras e troca de tarefas. }\end{array}$ \\
\hline $\begin{array}{l}\text { Cabanac et } \\
\text { al. (2013) }\end{array}$ & 128 & 432 & $\begin{array}{l}\text { Investigar se estudar } \\
\text { música a longo prazo } \\
\text { sistematicamente melhora } \\
\text { o desempenho académico. }\end{array}$ & $\begin{array}{l}\text { Resultados dos testes } \\
\text { com desempenho } \\
\text { quantificável, incluindo: } \\
\text { desporto, ciência, } \\
\text { matemática, francês, } \\
\text { inglês, história, química, } \\
\text { física, espanhol, ética, } \\
\text { mundo atual. }\end{array}$ & $\begin{array}{l}\text { Os alunos que selecionaram o curso de } \\
\text { música tiveram melhor desempenho do } \\
\text { que os que escolheram artes plásticas } \\
\text { ou artes dramáticas, apesar de todos } \\
\text { terem um desempenho inicial alto. } \\
\text { Os resultados confirmam que a música } \\
\text { tem uma ligação com a cognição. } \\
\text { Contudo, eles não indicam causalidade, } \\
\text { isto é, se os alunos têm melhores notas } \\
\text { porque praticam música ou se escolhem } \\
\text { música porque são melhores na escola. }\end{array}$ \\
\hline $\begin{array}{l}\text { Kaviani et } \\
\text { al. } \\
\text { (2013) }\end{array}$ & 30 & 30 & $\begin{array}{l}\text { Medir os efeitos da } \\
\text { formação musical } \\
\text { no desenvolvimento } \\
\text { cognitivo de crianças } \\
\text { em idade pré-escolar. }\end{array}$ & $\begin{array}{l}\text { Tehran-Stanford-Binet } \\
\text { Intelligence Scale (TSB); } \\
\text { 'Orff method'. }\end{array}$ & $\begin{array}{l}\text { A participação em aulas de música pode } \\
\text { conduzir a melhorias no funcionamento } \\
\text { intelectual e aumento das competências } \\
\text { verbais e não-verbais, bem como a um } \\
\text { aumento da capacidade de memória e de } \\
\text { desempenho. }\end{array}$ \\
\hline $\begin{array}{l}\text { Hyde et al. } \\
\text { (2009) }\end{array}$ & 15 & 16 & $\begin{array}{l}\text { Investigar mudanças } \\
\text { estruturais cerebrais } \\
\text { em relação a mudanças } \\
\text { comportamentais, em } \\
\text { crianças que tiveram } 15 \\
\text { meses de treino musical } \\
\text { relativamente a um grupo } \\
\text { de crianças que não } \\
\text { tiveram treino musical. }\end{array}$ & $\begin{array}{l}\text { Melodic and Rhythmic } \\
\text { Discrimination Test Battery; } \\
\text { Wechsler Intelligence Scale } \\
\text { for Children III (WISC-III); } \\
\text { Raven's Progressive Matrices } \\
\text { (RPM); } \\
\text { Auditory Analysis Test; } \\
\text { MRI Scanner. }\end{array}$ & $\begin{array}{l}\text { As crianças instrumentistas mostraram } \\
\text { mudanças comportamentais em apenas } \\
15 \text { meses na tarefa motora de dedo e } \\
\text { melodia/tarefas rítmicas, mas não nas } \\
\text { tarefas não musicais. Além disso, as crianças } \\
\text { instrumentistas mostraram maiores } \\
\text { mudanças, em comparação com o grupo } \\
\text { de controlo, em áreas motoras do cérebro, } \\
\text { como o giro pré-central direito (área da } \\
\text { mão motora), e no corpo caloso ( } 4 .^{\circ} \text { e } 5 .^{\circ} \\
\text { segmentos/metade do corpo), bem como } \\
\text { numa região auditiva (giro de Heschl). } \\
\text { Embora as diferenças cerebrais estruturais } \\
\text { fossem esperadas em áreas do cérebro } \\
\text { motoras e auditivas, diferenças significativas } \\
\text { cerebrais também foram encontradas em } \\
\text { várias áreas frontais, no peri-cingulado } \\
\text { posterior esquerdo e na região média do } \\
\text { occipital esquerdo. } \\
\text { O facto de não haver diferenças cerebrais } \\
\text { estruturais encontradas entre os grupos } \\
\text { antes do início do treino musical indica } \\
\text { que o desenvolvimento diferencial dessas } \\
\text { regiões cerebrais é mais induzido pela } \\
\text { prática instrumental do que pelos preditores } \\
\text { biológicos preexistentes da musicalidade. } \\
\text { Os resultados fornecem novas evidências de } \\
\text { plasticidade cerebral estrutural induzida por } \\
\text { treinamento na primeira infância. }\end{array}$ \\
\hline
\end{tabular}




\begin{tabular}{|c|c|c|c|c|c|}
\hline \multirow[t]{2}{*}{ Estudo } & \multicolumn{2}{|c|}{$\begin{array}{l}\text { Participantes } \\
(n)\end{array}$} & \multirow[t]{2}{*}{ Objetivo do estudo } & \multirow[t]{2}{*}{ Medidas de Avaliação } & \multirow{2}{*}{$\begin{array}{l}\text { Influência da Formação Musical } \\
\text { nos Objetivos em Estudo }\end{array}$} \\
\hline & GE & GC & & & \\
\hline $\begin{array}{l}\text { Forgeard } \\
\text { et al. } \\
\text { (2008) }\end{array}$ & 41 & 18 & $\begin{array}{l}\text { Verificar a associação } \\
\text { entre o treino } \\
\text { musical instrumental } \\
\text { na infância e os } \\
\text { resultados intimamente } \\
\text { relacionados com a } \\
\text { formação musical, } \\
\text { bem como aqueles } \\
\text { mais indiretamente } \\
\text { relacionados. }\end{array}$ & $\begin{array}{l}\text { Gordon's Intermediate } \\
\text { Measures of Music } \\
\text { Audiation (IMMA); } \\
\text { Tarefa de discriminação } \\
\text { melódica e rítmica; } \\
\text { Tarefa de aprendizagem } \\
\text { motora; } \\
\text { Wechsler Intelligence } \\
\text { Scale for Children III } \\
\text { (WISC-III); } \\
\text { Raven's Progressive } \\
\text { Matrices (RPM); } \\
\text { Auditory Analisis Test; } \\
\text { KeyMath-Revised: A } \\
\text { Diagnostic Inventory of } \\
\text { Essential Mathematics. }\end{array}$ & $\begin{array}{l}\text { O envolvimento musical foi relacionado } \\
\text { com as competências: motoras finas, } \\
\text { discriminação da melodia e verbais. } \\
\text { No entanto, não foi encontrada uma } \\
\text { relação com as competências espaciais, } \\
\text { a inteligência geral, a consciência } \\
\text { fonológica e a matemática. }\end{array}$ \\
\hline
\end{tabular}

A partir da revisão, verificou-se que quatro dos estudos mencionam uma influência positiva da formação musical (teórica e/ou instrumental) no desempenho académico em geral (Cabanac et al., 2013) ou em disciplinas tais como o Inglês (Yang et al., 2014) e a Matemática (Hallam \& Rogers, 2016; Nutley et al., 2014). Contudo, os estudos de Yang et al. (2014) e Forgeard et al. (2008) não confirmam os resultados acima mencionados quanto às competências matemáticas. No estudo de Yang et al. (2014), as crianças que estudaram música superaram os não-músicos só no desempenho musical e no desenvolvimento da língua inglesa. Contudo, e embora o treino musical pareça estar correlacionado com o desenvolvimento académico final das crianças na língua materna (chinês), na língua inglesa e na matemática, isso não contribuiu de forma independente para o desenvolvimento da língua materna ou das competências matemáticas. Também Forgeard et al. (2008) indicaram que as crianças que aprenderam a tocar um instrumento, durante 3 ou mais anos, superaram os seus pares de controlo em áreas estritamente relacionadas com a música - competências motoras finas e discriminação entre melodias -, mas não ao nível das competências fonémicas, espaciais ou da matemática. 0 grupo de controlo superou também no desempenho verbal (vocabulário) e no raciocínio não-verbal, tendo sido o treino musical apontado como um preditor destas capacidades.

Por sua vez, Hallam e Rogers (2016) referem ainda que os progressos superiores e melhores resultados académicos têm maior impacto nas crianças que tocam um instrumento há mais tempo.

Observou-se igualmente, nos estudos revistos, uma associação do treino musical a benefícios cognitivos, i.e., melhor desempenho nos testes de raciocínio, memória de trabalho visuoespacial (Jaschke et al., 2018; Nutley et al., 2014) e verbal ao longo do tempo (Nutley et al., 2014), velocidade de processamento (Nutley et al., 2014; Roden et al., 2014; Zuk et al., 2014), capacidades musicais e rítmicas, atenção visual e auditiva (Roden et al., 2014), aumento significativo do Quociente de Inteligência (QI), especificamente, no raciocínio verbal e memória a curto prazo (Kaviani et al., 2014). No raciocínio numérico e visual/abstrato as competências não diferiram entre o grupo que recebeu aulas de música e o que não recebeu (Kaviani el al., 2014). 
Não concordante com estes resultados, encontramos o estudo de D'Souza e Wiseheart (2018), em que não foram observados efeitos no desempenho cognitivo, em crianças com idades compreendidas entre os 6 e os 9 anos de idade, após um curto período de aprendizagem musical, em comparação com outros dois grupos (grupo de dança e grupo de controlo). Este estudo contribui para a tese de inconsistência de resultados, já que levanta a questão de se efetivamente o enriquecimento mental pode ocorrer na aprendizagem num curto prazo de tempo. Também não foram encontradas alterações nas competências cognitivas relacionadas com a aprendizagem musical (memória de trabalho, controlo de interferência, troca de tarefas, velocidade de processamento, vocabulário recetivo ou inteligência não verbal) após a intervenção experimental. Deste modo, a ausência de melhoria nas medidas cognitivas rejeita as propostas teóricas anteriores das competências cognitivas essenciais (funções executivas, velocidade de processamento), ou mecanismos de aprendizagem básicos (coordenação multimodal e entrainment do ritmo), como intermediários para um benefício cognitivo.

Também Zuk et al. (2014) focaram a relação entre o treino musical e uma maior ativação cerebral em regiões das Funções Executivas (FE) durante o paradigma de alternância de tarefas, descobrindo que crianças com uma média de 5.2 anos de treino musical revelaram um desempenho aprimorado em vários constructos das FE, em comparação com não-músicos, especialmente nas medidas de flexibilidade cognitiva, nos subtestes de fluência verbal e de Trail Making (através de tarefas de sequenciação visual-motoras), do Teste Delis-Kaplan Executive Function System (DKEFS) e velocidade de processamento, não existindo nenhuma diferença significativa na memória de trabalho entre os dois grupos em estudo. Para além disso, detetaram, através da investigação dos correlatos neuronais de alternância da atenção entre tarefas (switching), que houve uma maior ativação na Área Motora Suplementar (AMS) e no Córtex Ventrolateral Prefrontal (CVLPF) em crianças treinadas musicalmente em comparação com crianças sem treino, através de comparação direta do cérebro e análise de ROI (regions of interest). Estes resultados apoiam a hipótese de que o funcionamento executivo pode ser um dos mecanismos que medeiam o vínculo relatado entre o treino musical e as competências académicas, uma vez que as competências das FE e académicas surgem frequentemente correlacionadas. Neste mesmo estudo, os investigadores notaram ainda uma associação entre vários constructos das FE em crianças com diferentes intensidades de treino musical. No entanto, não foram observadas diferenças ao nível da inibição.

Jaschke et al. (2018) analisaram os efeitos das aulas de música estruturada nas subfunções executivas subjacentes ao desempenho académico em crianças do $1^{\circ}$ ciclo escolar. Os resultados mostraram que as crianças que participavam em aulas de música que seguiam uma estruturação e objetivo delineado tiveram um melhor desempenho em tarefas cognitivas, tais como o QI verbal, planeamento e inibição, quando comparadas aos controlos. Nas subfunções executivas primordiais para a perceção, processamento e execução da música também foram encontrados aumentos significativos na inibição e no planeamento, bem como no QI verbal. 
Nos estudos que investigaram mudanças estruturais cerebrais entre músicos e não-músicos foram observadas diferenças no volume de massa cinzenta no lobo temporal, situado principalmente nos giros fusiformes temporais e occipitais inferiores e na ínsula, caudado e putâmen, áreas relacionadas com a descodificação de notação musical (Nutley et al., 2014), e mudanças nas áreas cerebrais motoras e auditivas e várias áreas frontais, no peri-cingulado posterior esquerdo e na região occipital média esquerda em crianças, após 15 meses de treino musical (Hyde et al., 2009). No entanto, nenhuma destas mudanças foi correlacionada com melhorias no desempenho motor e capacidades auditivas (Hyde et al., 2009). Para além disso, no estudo de Hyde et al. (2009), apenas foi considerado o efeito da música no desenvolvimento do cérebro, sem ter em consideração o desempenho académico.

\section{DISCUSSÃO}

A presente revisão sistemática da literatura identificou 12 estudos empíricos publicados nos últimos 11 anos e que contribuem na reflexão sobre a influência da formação musical no desenvolvimento do raciocínio matemático; nas funções cognitivas (memória e atenção); e na plasticidade cerebral. Apesar de existirem estudos com foco no impacto da formação musical nas competências académicas e cognitivas em crianças e adolescentes, nenhum sintetizou diretamente os seus efeitos estritamente nas competências matemáticas.

O desempenho académico em crianças e/ou adolescentes em idade escolar, durante ou após exposição a um treino musical teórico e/ou instrumental, foi avaliado em cinco estudos (Cabanac et al., 2013; Forgeard et al., 2008; Hallam \& Rogers, 2016; Nutley et al., 2014; Yang et al., 2014). Contudo, se por um lado não há dados suficientes a suportar que a formação musical melhora as competências académicas, no geral, e as competências matemáticas, em específico (Forgeard et al., 2008; Yang et al., 2014), por outro não há também evidências a apoiar que o treino musical (teórico e/ou prático) não tem efeito nestas proficiências (Cabanac et al., 2013; Hallam \& Rogers, 2016; Nutley et al., 2014). A variabilidade de resultados pode ser atribuída aos diferentes modelos de estudo e diferentes instrumentos de avaliação utilizados. Para além disso, na matemática, cada estudo analisa um domínio diferente, podendo essa ser outra razão para não se encontrar consenso nos vários resultados (Cabanac et al., 2013; Forgeard et al., 2008; Hallam \& Rogers, 2016; Nutley et al., 2014). Um outro ponto de discussão será o período de intervenção, na medida em que pode ser um forte indicador de efeito ou da sua falta, uma vez que alguns dos estudos envolveram apenas intervenções educacionais com duração inferior a 1 ano (Forgeard et al., 2008; Kaviani et al., 2014).

No que diz respeito às funções cognitivas, os resultados obtidos nos estudos de Guo et al. (2018) e D’Souza e Wiseheart (2018) não são unânimes a identificar os benefícios cognitivos do treino musical, nomeadamente no que se refere à velocidade de processamento e à capacidade verbal. No estudo de Guo et al. (2018), os autores, após medirem as funções cognitivas antes e depois da aplicação de um programa de treino musical durante 6 semanas, apenas conseguiram observar uma melhoria na memória de trabalho 
de crianças entre os 6 e os 8 anos. D’Souza e Wiseheart (2018), para além de não observarem alterações nas competências cognitivas relacionadas com a aprendizagem musical, também não encontraram benefícios na memória de trabalho, ao contrário do que se verificou no estudo de Guo et al. (2018), na troca de tarefas, no controlo de interferência e na inteligência não-verbal. Ao nível do raciocínio, memória de trabalho visuoespacial e verbal, ao longo do tempo, e capacidades musicais e rítmicas, flexibilidade (ex.: fluência verbal), raciocínio verbal e memória a curto prazo, os estudos são coincidentes quanto à influência positiva que a formação musical exerce sobre estas funções (Jaschke et al., 2018; Kaviani et al., 2014; Nutley et al., 2014; Roden et al., 2014; Zuk et al., 2014). No que diz respeito à influência positiva da educação musical, a longo prazo, em competências cognitivas como a inibição, os resultados de Jaschke et al. (2018) contradizem os de Guo et al. (2018) e Zuk et al. (2014), tendo em consideração que em ambos os estudos não foram observados efeitos significativos.

Relativamente à atenção, Roden et al. (2014) mostram uma relação entre o treino musical e as capacidades de atenção auditiva. No entanto, estes resultados não são diretamente comparáveis com estudos que focam a relação entre o treino musical e a atenção visual.

Em dois estudos, Nutley et al. (2014) e Hyde et al. (2009), que avaliaram mudanças estruturais cerebrais entre crianças músicas e não-músicas, foram encontradas novas evidências para plasticidade cerebral estrutural induzida pelo treino musical na primeira infância. De acordo com Hyde et al. (2009), estas descobertas revelam que o treino musical pode em apenas 15 meses, na primeira infância, levar a mudanças cerebrais estruturais que divergem do desenvolvimento típico do cérebro, podendo a plasticidade ocorrer em regiões do cérebro que controlam funções primárias importantes para tocar um instrumento musical, e também em regiões do cérebro que podem ser responsáveis pelo tipo de integração sensoriomotora multimodal que provavelmente está subjacente à aprendizagem instrumental. Para além disso, o facto de não terem sido encontradas diferenças cerebrais estruturais entre os dois grupos em estudo, antes do início do treino musical, revelou que o desenvolvimento diferencial dessas regiões cerebrais é mais induzido pela prática instrumental do que pelos preditores biológicos preexistentes de musicalidade.

Na mesma sequência, Nutley et al. (2014) realçaram igualmente uma diferença na densidade de massa cinzenta no cérebro, em áreas relacionadas à descodificação de notação musical.

\section{CONCLUSÃO}

Apesar de já existirem estudos a analisar os efeitos da formação musical (teórica e/ou instrumental), a nível académico, cognitivo, estrutural e funcional do cérebro, poucos são os que demonstram a existência clara de uma relação entre a educação musical e as competências académicas, especialmente na matemática. No que se refere aos benefícios da primeira sobre a segunda, ainda não se verifica consenso na literatura. 
Com esta revisão sistemática é possível identificar a necessidade de utilização de métodos de investigação homogéneos, com recurso a testes padronizados, para a obtenção de resultados robustos que permitam ser incorporados de forma confiável neste domínio científico. 0 período de intervenção também deve ser tido em consideração, visto que poderá ser um forte indicador de um efeito positivo ou negativo. A intervenção deverá ter a duração de pelo menos 1 ano, de forma a mostrar resultados claros, sendo que estudos aleatórios controlados que se estendam por um período de tempo mais alargado, com diversas medições a partir da linha de base, tenderão a desenvolver um método de pesquisa mais fiável.

A par do aumento de estudos com foco no impacto da formação musical nas competências académicas e cognitivas, em crianças e adolescentes, será essencial a condução de mais investigações que isolem especificamente a componente matemática, de forma a poder medir os efeitos da formação musical diretamente nestas competências.

\section{REFERÊNCIAS}

Beer, M. (2005). Mathematics and music: Relating science to arts. East Coast College of English.

Cabanac, A., Perlovsky, L., Bonniot-Cabanac, M. C., \& Cabanac, M. (2013). Music and academic performance. Behavioural Brain Research, 256, 257-260. https://doi.org/10.1016/j.bbr.2013.08.023

Catterall,J., Chapleau, R., \& Iwanaga,J. (1999). Involvement in the arts and human development: General involvement and intensive involvement in music and theater arts. In E. Fiske (Ed.), Champions of change: The impact of the arts on learning (pp.1-18). Arts Education Partnership and President's Committee on the Arts and Humanities.

Costa-Giomi, E. (1999). The effects of three years of piano instruction on children's cognitive development. Journal of Research in Music Education, 47(3), 198-212. https://doi.org/10.2307/3345779

D’Souza, A. A., \& Wiseheart, M. (2018). Cognitive effects of music and dance training in children. Archives of Scientific Psychology, 6(1), 178-192. http://dx.doi.org./10.1037/arc0000048

Fitzpatrick, K. R. (2006). The effect of instrumental music participation and socioeconomic status on Ohio fourth-, sixth-, and ninth-grade proficiency test performance. Journal of Research in Music Education, 54(1), 73-84.

http://doi.org/10.1177/002242940605400106

Forgeard, M., Winner, E., Norton, A., \& Schlaug, G. (2008). Practicing a musical instrument in childhood is associated with enhanced verbal ability and nonverbal reasoning. PLoS ONE, 3(10), e3566. https://doi.org/10.1371/journal. pone.0003566

Grout, D. J., \& Palisca, C. V. (2014). História da música ocidental (6. ${ }^{\text {a ed.). }}$ Gradiva.

Guo, X., Ohsawa, C., Suzuki, A., \& Sekiyama, K. (2018). Improved digit span in children after a 6-week intervention of playing a musical instrument: An exploratory randomized controlled trial. Frontiers in Psychology, 8(2303). http://doi. org/10.3389/fpsyg.2017.02303 
Hallam, S., \& Rogers, K. (2016). The impact of instrumental music learning on attainment at age 16: A pilot study. British Journal of Music Education, 33(3), 247-261. https://doi.org/10.1017/S0265051716000371

Hyde, K. L., Lerch, J., Norton, A., Forgeard, M., Winner, E., Evans, A. C., \& Schlaug, G. (2009). Musical training shapes structural brain development. The Journal of Neuroscience, 29(10), 3019-3025. http://doi.org/10.1523/ JNEUROSCI.5118-08.2009

Jaschke, A. C., Honing, H., \& Scherder, J. A. (2018). Longitudinal analysis of music education on executive functions in primary school children. Frontiers in Neuroscience, 12(103). http://doi.org/10.3389/fnins.2018.00103

Jones, S. M., \& Zigler, E. (2002). The Mozart effect: Not learning from history. Journal of Applied Developmental Psychology, 23(3), 355-372. https://doi. org/10.1016/S0193-3973(02)00113-2

Kaviani, H., Mirbaha, H., Pournaseh, M., \& Sagan, O. (2014). Can music lessons increase the performance of preschool children in IQ tests? Cognitive Processing, 15, 77-84 http://doi.org/10.1007/s10339-013-0574-0

Kenealy, P., \& Monseth, A. (1994). Music and IQ tests. Psychologist, 7, 346.

Kravanja, P. (2008). Music: A mathematical offering. The Mathematical Intelligencer, 30(1), 76-77. http://doi.org/10.1007/BF02985765

Luís, C. (2013). A Aprendizagem musical como elemento de aperfeiçoamento de competências matemáticas (Tese de doutoramento não publicada). Universidade de Aveiro. https://ria.ua.pt/handle/10773/11448?locale=en

Miendlarzewska, E. A., \& Trost, W. J. (2014). How musical training affects cognitive development: Rhythm, reward and other modulating variables. Frontiers in Neuroscience, 7(279). http://doi.org/10.3389/fnins.2013.00279

Moreno, S., Bialystok, E., Barac, R., Schellenberg, E. G., Cepeda, N. J., $\&$ Chau, T. (2011). Short-term music training enhances verbal intelligence and executive function. Psychological Science, 22(11), 1425-1433. http://doi. org/10.1177/0956797611416999

Morrison, S. J. (1994). Music students and academic growth. Music Educators Journal, 81(2), 33-36. https://doi.org/10.2307/3398812

Moura, M. (2012). Projectos artístico-musicais: O contributo do ensino não formal para o desempenho académico e para a consideração das competências musicais no segundo ciclo do ensino básico (Tese de doutoramento não publicada). Universidade Católica Portuguesa. https://repositorio.ucp.pt/ handle/10400.14/12583

Newman, J., Rosenbach, J. H., Burns, K. L., Latimer, B. C., Matochia, H. R., \& Vogt, E. R. (1995). An experimental test of "The Mozart Effect": does listening to his music improve spatial ability? Perceptual and Motor Skills, 81, 1379-1387. https://doi.org/10.2466/pms.1995.81.3f.1379

Nutley, S., Darki, F., \& Klingberg, T. (2014). Music practice is associated with development of working memory during childhood and adolescence. Frontiers in Human Neuroscience, 7(296). https://doi.org/10.3389/fnhum.2013.00926

O'Hanlon, J. F. (1981). Boredom: Practical consequences and theory. Acta Psychologica, 49(1), 53-82. http://doi.org/10.1016/0001-6918(81)90033-0 
Pereira, S. (2012). Benefícios cognitivos do treino musical em jovens (Dissertação de mestrado não publicada). Universidade da Beira Interior. https://ubibliorum.ubi.pt/bitstream/10400.6/2647/1/Disserta\%C3\%A7ao-\%20 Beneficios\%20cognitivos\%20do\%20treino\%20musical\%20em\%20jove.pdf

Ramalho, J. (2014). A aprendizagem musical e escolar da criança: Contributo para uma relação de potencialidade (Tese de doutoramento não publicada). Universidade de Coimbra. https://estudogeral.sib.uc.pt/handle/10316/23720

Rauscher, F. H., Shaw, G. L., Levine, L. J., Ky, K. N., \& Wright, E. L. (1994). Music and spatial task performance: A causal relationship. Paper presented at the $102^{\text {nd }}$ Annual Meeting of the American Psychological Association, Los Angeles, CA.

Rauscher, F. H., Shaw, G. L., Levine, L. J., Wright, E. L., Dennis, W. R., \& Newcomb, R. L (1997). Music training causes long-term enhancement of preschool children's spatial-temporal reasoning. Neurological Research, 19(1), 2-8.

Roden, I., Könen, T., Bongard, S., Frankenberg, E., Friedrich, E. K., \& Kreutz, G. (2014). Effects of music training on attention, processing speed and cognitive music abilities - Findings from a longitudinal study. Applied Cognitive Psychology, 28(4), 545-557. http:// onlinelibrary.wiley.com/doi/abs/10.1002/acp.3034

Sanders, E. (2012). Investigating the relationship between musical training and mathematical thinking in children. Procedia - Social and Behavioral Sciences, 55(5), 1134-1143. https://doi.org/http://dx.doi.org/10.1016/j.sbspro.2012.09.607

Schellenberg, E. G. (2001). Music and nonmusical abilities. Annals of the New York Academy of Sciences, 930, 355-371. https://doi. org/10.1111/j.1749-6632.2001.tb05744.x

Schellenberg, E. G. (2011). Examining the association between music lessons and intelligence. British Journal of Psychology, 102(3), 283-302. http://doi. org/10.1111/j.2044-8295.2010.02000.x

Schlaug, G., Norton, A., Overy, K., \& Winner, E. (2005). Effects of music training on the child's brain and cognitive development. Annals of the New York Academy of Sciences, 1060(1), 219-230. http://doi.org/10.1196/annals.1360.015

Schneider, T. W., \& Klotz, J. (2000). The impact of music education and athletic participation on academic achievement. Paper presented at the annual meeting of the Mid-South Educational Research Association, Bowling Green, KY.

Southgate, D. E., \& Roscigno, V.J. (2009). The impact of music on childhood and adolescent achievement. Social Science Quarterly, 90(1), 4-21. http://doi. org/10.1111/j.1540-6237.2009.00598.x

Steele, K. M., Bass, K. E., \& Crook, M. D. (1999). The mystery of the Mozart Effect: failure to replicate. Psychological Science, 10(4), 366-369. https://doi. org/10.1111/1467-9280.00169

Stough, C., Kerkin, B., Bates, T., \& Mangan, G. (1994). Music and spatial IQ. Personality and Individual Differences, 17, 695. https://doi. org/10.1016/0191-8869(94)90145-7

Wetter, O. E., Koerner, F., \& Schwaninger, A. (2009). Does musical training improve school performance? Instructional Science, 37(4), 365-374. http://doi. org/10.1007/s11251-008-9052-y

Wilson, T. L., \& Brown, T. L. (1997). Reexamination of the effect of Mozart's music on spatial-task performance. The Journal of Psychology, 131(4), 365-370. https://doi.org/10.1080/00223989709603522 
Wright, D. (2009). Mathematics and music. American Mathematical Society. Yang, H., Ma, W., Gong, D., Hu, J., \& Yao, D. (2014). A longitudinal study on children's music training experience and academic development. Scientific Reports, 4, 5854. https://doi.org/10.1038/srep05854

Zuk, J., Benjamin, C., Kenyon, A., \& Gaab, N. (2014). Behavioral and neural correlates of executive functioning in musicians and non-musicians. PLOS ONE, 9(6), e99868. http://doi.org/10.1371/journal.pone.0099868 
i Universidade Católica Portuguesa, Instituto de Ciências da Saúde, Portugal. https://orcid.org/0000-0003-3174-6641

ii Universidade Católica Portuguesa, Instituto de Ciências da Saúde, Centro de Investigação Interdisciplinar em Saúde, Portugal. https://orcid.org/0000-0002-7298-4892

iii Universidade Católica Portuguesa, Instituto de Ciências da Saúde, Centro de Investigação Interdisciplinar em Saúde, Portugal. https://orcid.org/0000-0002-9148-3719

Toda a correspondência relativa a este artigo deve ser enviada para:

Susana Azevedo

Instituto de Ciências da Saúde

Universidade Católica Portuguesa

Palma de Cima

1649-023 Lisboa, Portugal

azevedosusana@gmail.com 


\title{
Contribution of musical training in the academic and cognitive performance of children and adolescents: A systematic review
}

\begin{abstract}
This systematic review aimed to analyze the recent literature on the influence that the study of music can exert on academic performance in general and on mathematics; in cognitive functions; and in brain plasticity. The review includes studies published between 2007 and 2018 in the PubMed and Complementary Index databases, Academic Search Complete, Education Source, Psychology and Behavioral Sciences Collection, Science Direct and PsycArticles, using the descriptor musical training, combined with descriptors linked to academic performance in general and in mathematics - academic achievement, mathematics and academic development -, and cognitive development - brain development and cognitive development. The studies were selected according to the following criteria: studies (i) published in peer-reviewed scientific journals, (ii) with children and adolescents up to 18 years of age, (iii) which included musical training in its theoretical and/or instrumental components. The relationship between musical education and academic performance in general and mathematics was inconsistent, and there was no consensus in the literature about the benefits of the former over the latter. Cognitive benefits and evidence for structural brain plasticity induced by early childhood music training were found, taking into account differences in gray matter volume.
\end{abstract}

Keywords: Musical training; Academic performance; Mathematical skills; Cognitive development. 


\section{Contribution de la formation musicale à la performance académique et cognitive des enfants et des adolescents: Une revue systématique}

\section{RÉSUMÉ}

Cette revue systématique visait à analyser la littérature récente sur l'influence que l'étude de la musique peut exercer sur les performances académiques en général et sur les mathématiques; dans les fonctions cognitives; et dans la plasticité cérébrale. La revue comprend des études publiées entre 2007 et 2018 dans les bases de données PubMed et Complementary Index, Academic Search Complete, Education Source, Psychology and Behavioral Sciences Collection, Science Direct et PsycArticles, en utilisant le descripteur de musical training, associé à des descripteurs liés aux performances académiques en général et en mathématiques - academic achievement, mathematics et academic development - et au développement cognitif - brain development et cognitive development. Les études ont été sélectionnées selon les critères suivants: études (i) publiées dans des revues scientifiques évalué par les pairs, (ii) avec des enfants et des adolescents de moins de 18 ans, (iii) qui comprenait une formation musicale dans ses composantes théoriques et/ou instrumentales. La relation entre l'éducation musicale et les performances académiques en général et les mathématiques était incohérente, et il n'y avait pas de consensus dans la littérature sur les avantages des premiers par rapport aux seconds. Des avantages cognitifs et des preuves de la plasticité structurelle du cerveau induite par la formation musicale au cours de la petite enfance ont été découverts, en tenant compte des différences de volume de matière grise.

Mots-clés : Formation musicale; Performance académique; Compétences en mathématiques; Développement cognitif. 